\title{
Estándares y trabajo colaborativo como parte de la enseñanza BIM en educación superior
}

\author{
doi: 10.33264/rpa.202001-12 \\ Karen Gutiérrez D. \\ Paulina Godoy del C. \\ Escuela de Arquitectura. Facultad de Arquitectura, Diseño y Artes.
}

\section{Resumen}

El documento se propone determinar la importancia de los espacios de colaboración en entornos BIM (Building Information Modeling) como fundamento del cambio de paradigma que vive hoy la educación superior, frente a la formación de profesionales con las competencias necesarias para responder a los requerimientos actuales y futuros de la industria AEC (Architecture, Engineering and Construction).

Para lograrlo, se revisarán los aspectos más relevantes a considerar para generar un cambio en la forma de enseñar, enfocándose en incentivar el uso de espacios de colaboración como parte de un proceso de trabajo multidisciplinario. En este sentido, la importancia de potenciar la enseñanza de la metodología BIM radica en su base de procesos y tecnologías colaborativas para aumentar la eficiencia en la ejecución de proyectos de la industria de la construcción. De esta forma se buscar fortalecer la respuesta académica a la disponibilidad de profesionales con las competencias exigidas en el mercado actual y presentar nuevas formas de hacer educación frente a una acelerada transformación digital. Además, se abordan los distintos estándares de la industria de la construcción, y en especial los que dan sustento a la metodología BIM, y que posibilitan mantener consistencia y trazabilidad de la información en todo el ciclo de vida de un proyecto, sin importar en número de participantes de distintas disciplinas que se involucren en él.

Palabras claves: Trabajo colaborativo, Industria 4.0, Transformación digital, BIM.

\section{Abstract}

This document seeks to establish the importance of collaborative work in Building Information Modeling (BIM) environments, as a foundation of the paradigm shift that is occurring in higher education, in training professionals with the necessary competences to meet the requirements of today's AEC industry.

To accomplish this, a revision is made of important aspects worth considering, about the necessity of transforming the present state of education to encourage the use of collaborative spaces as a part of a process of multi-disciplinary work. In this sense, 
the importance of promoting the teaching of the BIM methodology lies in its base of collaborative processes and technologies to increase efficiency in the execution of projects in the construction industry. In this way, this document seeks to strengthen the academic response to the availability of professionals with the skills required in the current market, and to present new ways of educating in the face of an accelerated digital transformation. In addition, the different Standards of the construction industry are addressed, and especially those that support the BIM methodology, and that make it possible to maintain consistency and traceability of information throughout the life cycle of a project, regardless of number of participants from different disciplines involved in it.

Keywords: Collaborative work, Industry 4.0, Digital transformation, BIM.

\section{Introducción}

La industria AEC está sufriendo uno de sus mayores cambios desde la industrialización. Klaus Schwab en su libro La cuarta revolución industrial, hace referencia a este cambio, la llamada revolución tecnológica cuyo impacto se estima significará una transformación de la humanidad. Ha cambiado la forma en que nos comunicamos, trabajamos y nos relacionamos, aspectos en los cuales ahora prima la velocidad, la información y las tecnologías que permiten estos intercambios.

El surgimiento de nuevos avances tecnológicos, como la robótica, el internet de las cosas (IoT), la impresión 3D, la nanotecnología, la inteligencia artificial entre otras, son cada vez más frecuentes, lo que implica un cambio constante en nuestra forma de vivir. Estas transformaciones también se dan en el ámbito profesional. En cuanto a las distintas industrias, esto se ve reflejado en aspectos como la aparición de nuevos modelos de negocios, transformación de los sistemas de producción y de entrega, por nombrar algunos. La industria $\mathrm{AEC}$ no es la excepción, sumando importantes cambios en su manera de operar en las últimas décadas. Enfrentando la baja productividad y poca eficiencia en los procesos involucrados en todas las etapas de un proyecto constructivo. De este modo y ya hace un tiempo, surge el concepto de Building Information Modeling (BIM), que consiste en "un conjunto de metodologías, tecnologías y estándares que permiten diseñar, construir y operar una edificación o infraestructura de forma colaborativa en un espacio virtual" 64.

Para comprender cómo se articula la metodología BIM es necesario entender los

\footnotetext{
64 Definición entregada por Planbim de Corfo en el Estándar BIM para Proyectos Públicos, basada en el BIM Dictionary de Bilal Succar , https://bimdictionary.com/en/building-information-modelling/1/
} 
conceptos que la determinan. En este sentido Bilal Succar ${ }^{65}$ menciona los conceptos fundamentales o "campos BIM" que sustentan el trabajo mediante esta metodología. En el campo de las tecnologías se tienen todas las herramientas, espacios virtuales de colaboración y trabajo en la nube, entre otras, que sustentan otros aspectos de esta nueva forma de trabajo colaborativo.

En el campo de los procesos encontraremos a las personas como actores participantes de las distintas etapas del desarrollo de un proyecto, desde su concepción inicial a su operación y mantenimiento. En el campo de las políticas encontraremos las instituciones regulatorias, académicas, centros de investigación y otros encargados de generar los lineamientos, estándares, indicadores y acuerdos contractuales.

De este modo es posible observar y comprobar que como base fundamental de un trabajo colaborativo en BIM, está fundado en los procesos, políticas y tecnologías.

Desde una mirada nacional y complementando la incorporación del trabajo colaborativo en las fuentes de trabajo futuras, en Mibim, "Matriz de Madurez de Implementación BIM para Organizaciones" 66 desarrollada por Planbim de Corfo, a la teoría anterior señalada se suma un cuarto pilar fundamental, las personas, que permite definir la estructura organizacional desde la mirada de los roles y perfiles necesarios para desarrollar las acciones vinculadas a BIM.

Desde este pilar se incorpora el desarrollo de capacidades relacionadas a la metodología ${ }^{67}$ desarrollados a partir de la "Matriz de Roles BIM", que definen cinco roles, nombrados de acuerdo con las responsabilidades BIM. De este modo se permite formular un círculo completo de conceptos que llegan a concretar el desarrollo de una metodología colaborativa en toda una organización.

En el siguiente esquema se explica la relación de los conceptos anteriormente señalados y cómo desde el pilar de las personas cobra protagonismo la academia como parte fundamental de las acciones que potencien estos espacios de trabajo colaborativos.

\footnotetext{
65 https://bimexcellence.org/projects/macro-adoption/

66 https://planbim.cl/test-matriz/

67 https://planbim.cl/biblioteca/documentos/

Revista Pensamiento Académico de la Universidad UNIACC

Vol. $3 \mathrm{~N}^{\circ} 1,2020$
} 
Imagen 01. Conceptos BIM y educación superior.

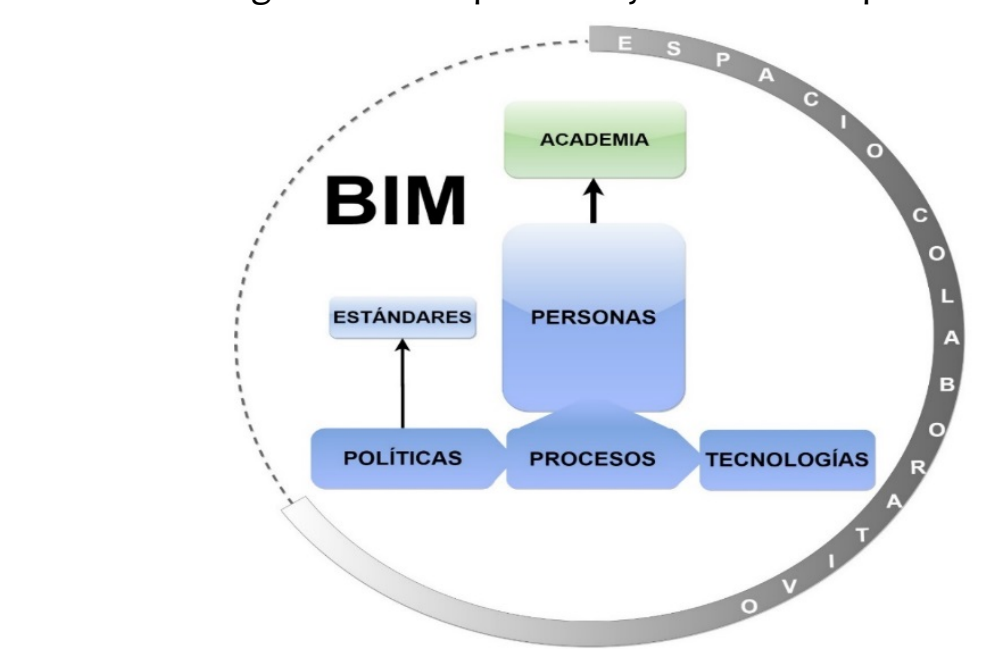

Fuente: Creación Paulina Godoy y Karen Gutiérrez, Santiago de Chile, 2020.

\section{Objetivo General}

Se han definido el siguiente objetivo general para establecer la línea de desarrollo del documento:

- Evidenciar la importancia de la metodología BIM como parte de los procesos de aprendizaje de los alumnos en carreras relacionadas a la industria AEC.

Para esto se han determinado objetivos específicos los cuales vienen a responder y complementar el proceso de investigación del documento:

- Comprender el contexto profesional real que enfrentarán los estudiantes al egresar, y las competencias que deben desarrollar como parte del perfil de egreso.

- Exponer la importancia de promover en la educación superior, los estándares vigentes y aplicables en la industria AEC.

- Entender la importancia de promover el trabajo colaborativo en educación superior.

- Reconocer los distintos estándares BIM que sustentan el trabajo colaborativo en la industria AEC.

\section{Metodología}

Este trabajo se ha desarrollado bajo la observación y análisis docente, obtenidos desde experiencias vertidas en el aula de clases derivadas en las asignaturas de Taller de Arquitectura de la carrera de Arquitectura UNIACC y del Magíster BIM de la misma casa de estudios. 
La primera etapa consistió en hacer una revisión y estudio de la formación actual de los alumnos que conforman la carrera de pregrado de Arquitectura y el postgrado Magíster BIM, y cómo estos dos programas académicos pudieran potenciar las competencias de los profesionales egresados. Luego, se desarrolló un espacio de discusión de las observaciones recolectadas entre las docentes autoras, como segundo método de acercamiento al tema tratado.

En cuanto a la transformación tecnológica que atraviesa la industria AEC, se decide plantear un estudio basado en la teoría y revisión de contenidos. De esta forma se busca obtener un marco de referencia que fundamente la incorporación del trabajo colaborativo y los estándares BIM como parte importante del currículo en carreras universitarias relacionadas a la industria AEC.

El resultado expuesto en este artículo responde al trabajo de revisión de la literatura actual respecto a BIM, y a la evaluación de distintas teorías de entidades nacionales e internacionales que articulan y difunden esta metodología. Además, se hace referencia al quehacer docente individual de cada una de las autoras respecto a los temas tratados, en directa relación con el desarrollo y proceso de aprendizaje de los alumnos.

\section{Cómo abordar BIM desde la academia}

Incluir o no la metodología BIM en la enseñanza de la Arquitectura, es una forma de entender o responder al mercado profesional y lo que está requiriendo. Muchas universidades a nivel internacional ya la han incorporado a sus mallas curriculares como una línea transversal de aprendizaje. A nivel nacional ha ido en aumento el número de carreras de pregrado que están relacionadas al área y que han incorporado a sus asignaturas optativas temáticas relacionadas a BIM. Según información extraída desde el Estudio de Identificación de Demanda de Capital Humano con Capacidades BIM en la Industria de la Construcción ${ }^{68}$, al 2017 del total de los programas de pregrado de la carrera de Arquitectura, un 64,5 \% ya habían incorporado BIM dentro de su currículo, viéndose ese porcentaje aumentado en los últimos años. En universidad UNIACC, la metodología BIM está integrada a la malla curricular de manera obligatoria y desde los primeros semestres de la carrera de Arquitectura.

Por otro lado, la oferta de postgrados y cursos de capacitación está mucho más

68IALE. Diciembre del 2017. "Estudio de Identificación de Demanda de Capital Humano con Capacidades BIM en la Industria de la Construcción.”, desarrollado para Planbim de Corfo. Santiago, 2017. https://planbim.cl/biblioteca/documentos-informes-estudios/ 
desarrollado, y es más frecuente ver programas de estudios relacionados a esta metodología, en especial diplomados y cursos específicos dedicados al uso de herramientas BIM. En este sentido, la Universidad UNIACC es la única casa de estudios que desde el año 2016 tiene un magíster específico del área, y en modalidad semipresencial, lo que permite cursarlo de manera online y desde cualquier parte del país, incluso del mundo.

Estos programas de estudios en general están orientados a todo profesional de la construcción: arquitectos, ingenieros, constructores, gerentes de proyectos y contratistas, que deseen organizar su equipo mediante un trabajo colaborativo apoyado en herramientas tecnológicas, asegurando la trazabilidad de la información.

El cómo poder abordar BIM desde la academia dependerá de los objetivos que se espera lograr de su enseñanza. El enfoque más frecuentemente observado es introducir a los alumnos a la metodología incorporando el uso de herramientas tecnológicas que la sustentan y como asignatura optativa. En este documento se propone, además, poner énfasis en dos conceptos fundamentales que permiten que el uso de las herramientas tecnológicas tenga reales beneficios, y ya se pueda hablar de método en función de hacer eficiente los procesos. Estos conceptos son los entornos de colaboración o entornos comunes de datos, y la estandarización de procesos e información.

En la medida que la academia potencie el trabajo colaborativo como un método de aprendizaje sustentado en hacer eficientes los procesos partir de las herramientas tecnológicas adecuadas, se desarrollarán las competencias necesarias conformes a lo que el mercado laboral espera.

\section{Contexto actual en la construcción}

BIM es una metodología que permite generar y gestionar de forma eficiente y trazable la información de un activo durante todo su ciclo de vida. Esta información se crea y desarrolla en un modelo virtual e inteligente, que contiene una base de datos actualizados en tiempo real con cada cambio que se efectúa en el proyecto. A su vez, este diseño tridimensional permite evaluar y previsualizar diversas soluciones de manera simultánea y analizar aspectos más allá de la forma, reduciendo errores y aportando información para la toma de decisiones. Los beneficios obtenidos de este proceso son muchos, y son más evidentes en la etapa operativa del edificio.

Al trabajar mediante la metodología BIM, las etapas de diseño, construcción y operación, se desarrollan en un entorno en común virtual, traspasando y enriqueciendo la información del proyecto hasta el fin de la vida útil del activo. Esto permite que, al requerirse realizar un cambio de uso o funcionalidad para darle una 
nueva vida al edificio, sea factible obtener información confiable de forma rápida desde el entorno común de datos y el modelo BIM.

En la actualidad, las etapas de diseño de un proyecto son más bien ejecutadas de una manera apresurada y bajo la marcha, lo que resulta en conflictos, interferencia en las especialidades, lo que afecta directamente en los tiempos y costos del proyecto. Los profesionales que intervienen en un proyecto realizado en BIM tanto en su coordinación como en el análisis y gestión, pueden llegar a reducir tiempos y costos, gracias a que esta metodología les da la posibilidad de detectar errores en la etapa de diseño, evitando que dichos errores pasen a la etapa de construcción.

Si observamos el panorama nacional, en Chile la metodología está mucho más internalizada. Esto ya que, con el objetivo de incrementar la productividad en la industria de la construcción, en el año 2016 se crea Planbim como iniciativa del estado, parte del Programa Estratégico de Productividad y Construcción Sustentable, Construye 2025. De este modo se ha buscado potenciar y promover la utilización de la metodología BIM para el desarrollo y operación de proyectos de edificación e infraestructura pública. Uno de los hitos de esta implementación fue el lanzamiento del Estándar BIM para Proyectos Públicos el año 2019. Esta iniciativa nacional, al promover y apoyar el uso de la metodología en las instituciones públicas, ha provocado una alta demanda de profesionales capacitados y con competencias en BIM, forzando a la academia a replantear el currículum de educación superior de las carreras relacionadas a la industria, y a la capacitación y adaptación de aquellos profesionales que llevan años de egreso y experiencia laboral.

\section{Proceso de trabajo en BIM}

Un proyecto de construcción debe observarse desde su etapa de planificación hasta su etapa operacional, como un proceso continuo que comprende todo el ciclo de vida del activo. De esta manera es posible evidenciar y comprender lo fundamental que es comprender la trazabilidad de la información que éste tiene y cómo va evolucionando en el desarrollo. El siguiente esquema explica las fases del ciclo de vida de un proyecto a nivel general. 
Imagen 02. Fases del ciclo de vida del proyecto y subfases.

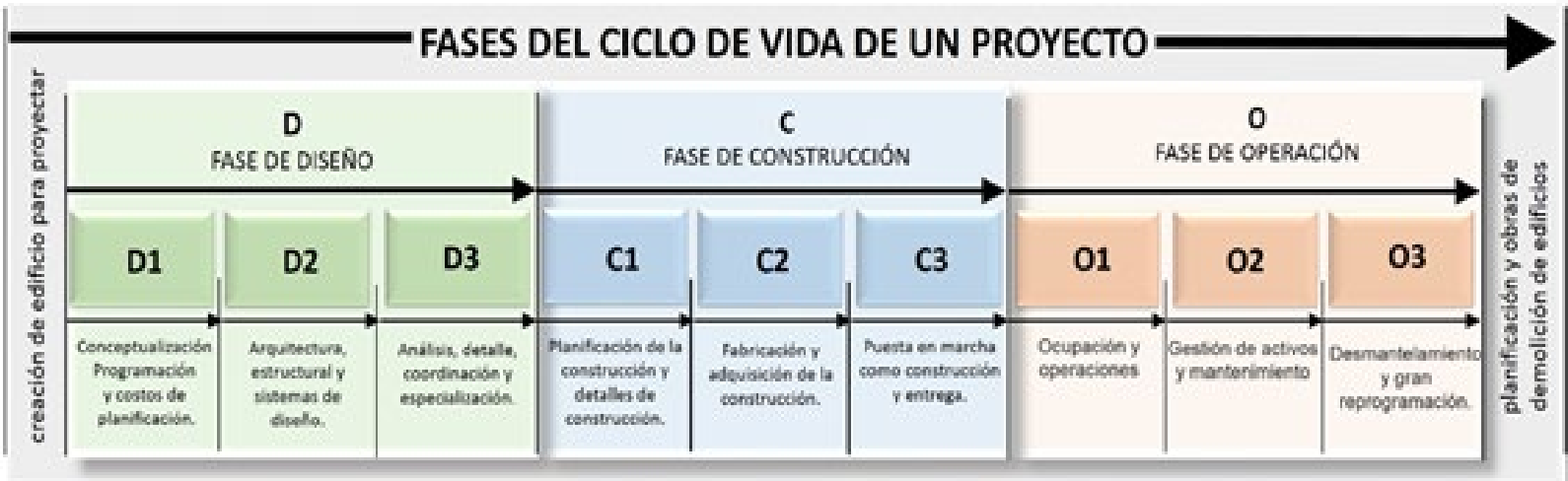

Fuente: Karen Gutiérrez, 2018. Basado en Succar, 2008

Para esto, BIM aporta desde la aplicación de tecnologías que permiten registrar la información de un activo en cada una de estas etapas, optimizando el traspaso y gestión de la información de manera fluida, de tal forma que el trabajo colaborativo sea eficaz y eficiente.

Un proyecto desarrollado de manera tradicional está definido por distintas etapas que se ejecutan de manera no colaborativa y no necesariamente manteniendo comunicación entre las partes. La información que se va generando desde una etapa no es siempre la misma que se pasa a la siguiente, produciendo errores, pérdidas y obsolescencia de los datos, aumentando tiempos y costos, los que impactan fuertemente en la etapa de construcción. Para lograr un trabajo integral, es necesario considerar la cantidad de profesionales que intervienen en un proyecto, y todas las variables que permiten una buena coordinación entre ellos. Desde aquí cobra relevancia la formación profesional y la importancia de fortalecer la adquisición de competencias y desarrollo de capacidades en entornos de colaboración, dado que sus funciones no serán ejecutadas de manera aislada, sino en ambientes multidisciplinarios. A continuación, se presenta un esquema que compara etapas de un proyecto en función de la madurez de BIM que tenga la organización. Se puede observar que en la etapa 1 de BIM se tiene un proceso de trabajo menos fluido, dada la escasa colaboración en cuanto al proceso de traspaso de información, mientras en la medida que se va haciendo este proceso colaborativo y trasversal en las etapas 2 y 3 se realiza de manera más eficiente. 
Imagen 03. Efecto de BIM en las fases del ciclo de vida del proyecto

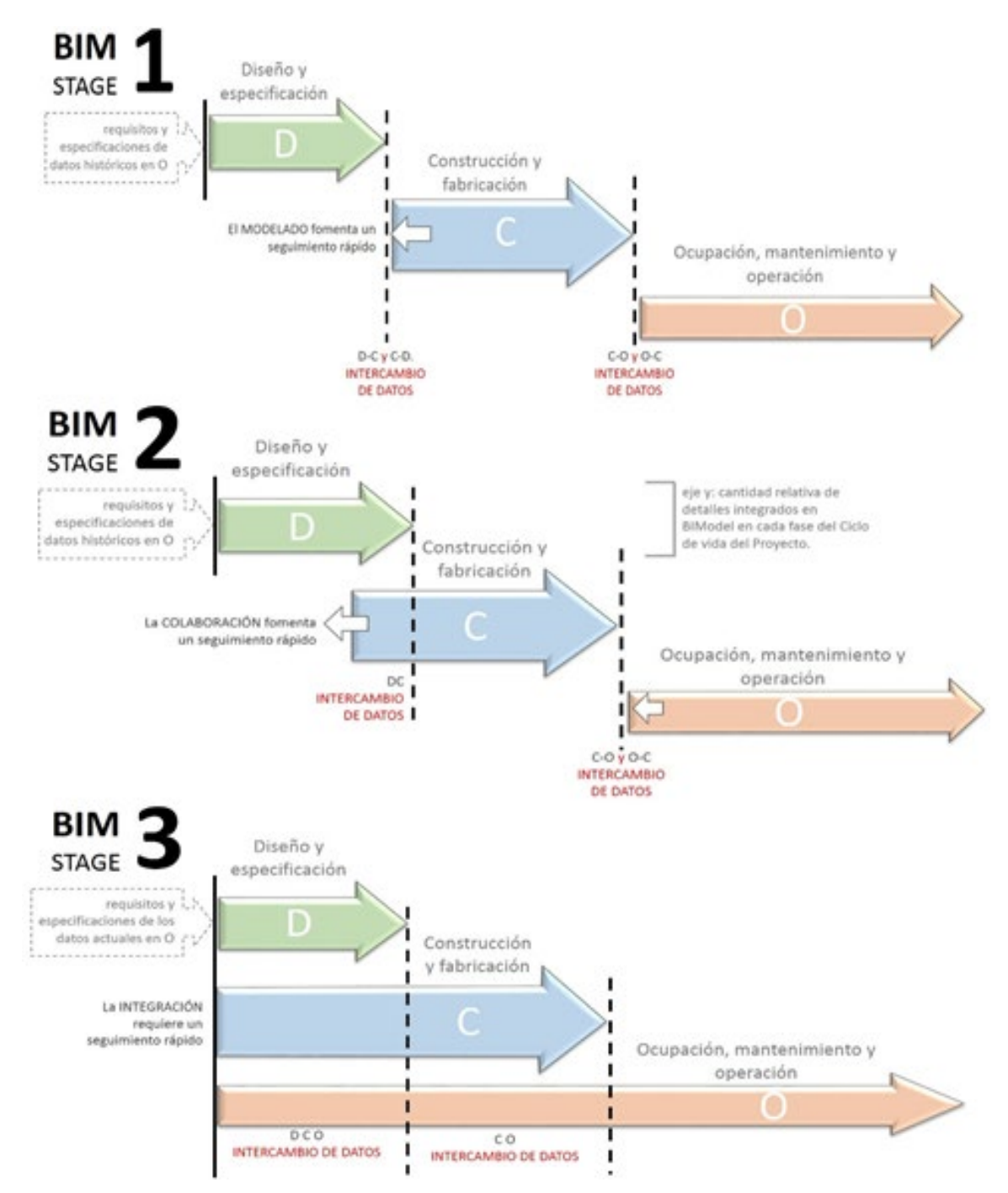

Fuente: Karen Gutiérrez, 2018. Basado en Succar, 2008.

Los niveles de madurez del BIM pueden estar medidos desde distintas matrices. A nivel nacional, existe la "Matriz de Madurez de Implementación BIM para organizaciones", Mibim, una herramienta interactiva online que permite a las empresas o instituciones hacer una autoevaluación respecto de su nivel de adopción de la metodología. En esta matriz se busca el avance en torno a 03 conceptos: planificar, implementar y mantener, y esto a su vez se evalúa en función de los 04 pilares, :estrategia, procesos, personas y tecnología. Por otro lado, y a nivel internacional, podemos mencionar, la "Matriz de Madurez BIM", BIm ${ }^{3}{ }^{69}$ que es una herramienta de conocimiento para medir la madurez 
de BIM en organizaciones y equipos de proyectos, basada en 02 ejes principales: conjunto de capacidades BIM y el índice de Madurez BIM.

\section{Trabajo colaborativo en entornos virtuales}

La gestión de la información en el desarrollo de un proyecto de la industria de la construcción es un proceso complejo que involucra gran cantidad de documentos y múltiples participantes de diversas áreas. Por esta razón, bajo la metodología BIM se plantea la utilización de los llamados Entornos Comunes de Datos o CDE (Common Data Environment). Estos son espacios digitales compartidos donde se almacena e intercambia la información del proyecto, al cual todos los integrantes de los equipos de trabajo tienen accesos definidos, en función del rol y responsabilidad que tengan. Para utilizar estos CDE se establecen estrategias de colaboración que permiten tener trazabilidad de la información, lo que a su vez permite a los participantes del proyecto tomar decisiones de manera oportuna.

Según lo expuesto por el Estándar BIM para Proyectos Públicos de Planbim, el CDE debe ser la única fuente de información, permitir recopilar, gestionar y difundir documentos y modelos que se utilicen en un proyecto, disponibilizando la información mediante un proceso estandarizado. La norma ISO 19650-1:2018 menciona que un CDE debe ser aquel espacio que permite la seguridad en el intercambio de información, brindando un flujo de trabajo colaborativo e interdisciplinario. Un CDE puede estar compuesto por más de una solución informática, dependiendo de la etapa del proyecto, los actores involucrados, las especialidades, entre otros aspectos. Además, el Estándar BIM para Proyectos Públicos de Planbim establece que estos sistemas deben considerar 03 condiciones: Una plataforma de colaboración que permita trabajar con información unificada y centralizada, una plataforma de gestión documental que permita controlar los procesos de intercambio de información, y formatos de requerimientos de información y colaboración que permitan el registro y trazabilidad de comentarios, incidencias y revisiones.

Cuando hablamos de trabajo colaborativo pareciera ser algo inherente en los proyectos de construcción, dada la cantidad de profesionales que intervienen en un proyecto. Eastman menciona acerca del trabajo colaborativo, "Es necesario un trabajo en conjunto y un espacio de colaboración, donde cada área pueda contribuir con los datos necesarios para lograr un objetivo en común" (Eastman, 2008). Pero ¿es suficiente establecer una base tecnológica para obtener beneficios del trabajo colaborativo? Los entornos virtuales de intercambio de información existen hace varios años, pero suelen ser mal utilizados lo que neutraliza las posibles mejoras en cuanto a la trazabilidad y confiabilidad de la información que su implementación 
debería conllevar. El componente humano y la estandarización de los procesos son fundamentales para aprovechar todo el potencial de estas herramientas tecnológicas. De esta manera comprendemos la importancia de entender que, si bien un CDE es una herramienta tecnológica, son los estándares quienes vienen a definir como es este traspaso de información, lo cual llega a concretarse a partir de las capacidades BIM del equipo de trabajo y los procesos definidos en torno a ello.

\section{Importancia de la estandarización en la metodología BIM}

Como se menciona anteriormente, para que el flujo de información se produzca de manera eficiente y que involucre a todos los actores relevantes de un proyecto, es fundamental el uso de un CDE como determinar qué información se compartirá, en qué momento, y cómo se realizará este proceso. Para establecer claramente estos puntos se desarrollan estándares. La International Organization for Standardization (ISO) explica que un estándar es un "Documento, establecido por consenso y aprobado por un organismo reconocido que entrega, para usos comunes y repetidos, reglas y directrices o características para actividades o sus resultados, ayudando a la obtención de un grado óptimo de ordenamiento en un contexto dado"70.

La importancia de implementar estándares de trabajo radica en que estos permiten establecer:

- Consenso entre distintas partes. La elaboración de un estándar debe incorporar la mayor variedad de actores involucrados en los procesos que se busca estandarizar. Esto no sólo tiene como beneficio el integrar las distintas visiones sobre el tema, evitando sesgos de unilateralidad, sino que también otorga una validación intrínseca al proceso de desarrollo del estándar.

- Lenguaje común. La diversidad de herramientas digitales y metodológicas, disciplinas e incluso nacionalidades y lenguas involucradas en los proyectos de cualquier industria en la actualidad, hacen necesario establecer acuerdos básicos y una terminología comprendida por todos los participantes sin ambigüedades.

- Trazabilidad de la información. El establecer con claridad los flujos de información desde las primeras fases de desarrollo de un proyecto contribuyen a no perderle el rastro en ningún momento a los datos generados y compartidos. Además, esto permite identificar a los responsables de generar, capturar,

70 Estándar BIM para Proyectos Públicos, Planbim, Santiago de Chile. 
compartir y gestionar la información, junto con las etapas del proyecto en las que se debe entregar o recibir los datos. Si consideramos el volumen de información involucrado en un proyecto desarrollado bajo la metodología BIM, se hace evidente la importancia de la transparencia en los procesos de manejo de datos y las distintas partes involucradas.

\section{Estándares BIM}

\subsection{Estándares abiertos}

Un estándar se puede llamar abierto cuando no tiene restricciones de utilización, está disponible públicamente, y se desarrolla en base al consenso y evaluación pública. La implementación de estos estándares permite a empresas de distintos tamaños trabajar con BIM sin depender exclusivamente de un solo fabricante de software. Esto incentiva mayor competencia, ampliando la oferta de proveedores de servicios. Además, facilitan la reutilización de la información y la integración con datos provenientes de industrias relacionadas. De este modo es posible decir que los estándares abiertos son parte fundamental del trabajo colaborativo, transparente y eficiente. A continuación, se mencionarán algunos.

IFC (Industry Foundation Classes). Estándar abierto para la especificación e intercambio de información de la industria de la construcción. Facilita la comunicación y colaboración entre los distintos especialistas involucrados en un proyecto de la industria AEC, sin importar la disciplina que practican, la fase del proyecto en la que se encuentran, y la herramienta tecnológica utilizada. En efecto, IFC es el estándar abierto internacionalmente más reconocido y utilizado en proyecto ejecutados con BIM. La entidad detrás de este desarrollo es la buildingSMART, que es un foro internacional enfocado en resolver los desafíos de la industria de la construcción digital, habilitando flujos de trabajo que fomentan la interoperabilidad entre las distintas aplicaciones de software existentes. En el año 2005, la versión 2x3 de este estándar fue certificado como norma PAS/ISO 16739-1, y la versión IFC4 se certificó en primera instancia en el 2013 y luego en 2018. 


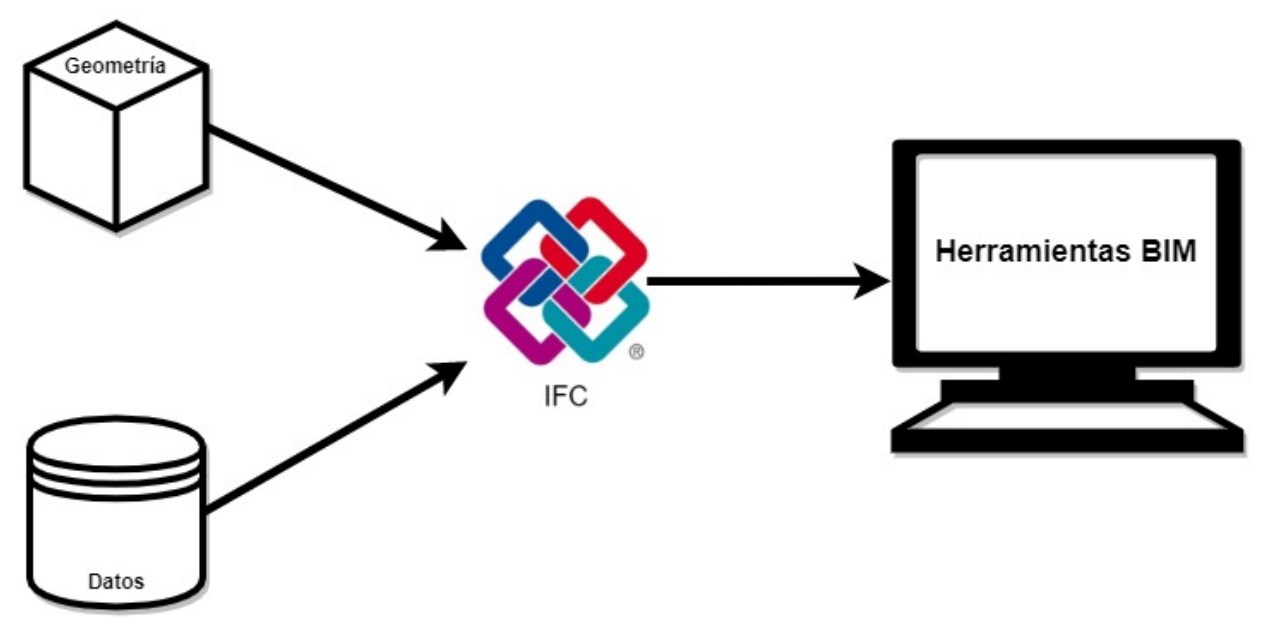

Fuente: Creación Paulina Godoy y Karen Gutiérrez, Santiago de Chile, 2020.

El esquema anterior muestra globalmente cómo, mediante IFC y su esquema de datos, se dispone de un lenguaje común, ordenado y estructurado, que permite que la información de un proyecto sea compartida e interpretada de una sola manera por los múltiples participantes que se involucren en cualquiera de sus etapas.

Este no es el único estándar desarrollado por buildingSMART. Esta institución también es responsable de desarrollar y mantener otros que se mencionan a continuación.

MVD - Model View Definitions: Un Model View Definition es un subconjunto de información del esquema general IFC para un uso o flujo de trabajo específico, acotando el alcance dependiendo de las necesidades del receptor de la información ${ }^{71}$. El hecho de filtrar la cantidad de información compartida tiene como objetivo comprender que cada especialidad necesita datos particulares para ejecutar su trabajo. La solución propuesta por buildingSMART evita un trabajo manual de seleccionar uno a uno los datos compartidos con cada participante del proyecto, estandarizando esta selección a casos particulares. 
BCF - BIM Collaboration Format: Este estándar tiene como objetivo facilitar el intercambio de comentarios entre distintos participantes de un proyecto, en base a archivos de modelo IFC, unificando la forma de comunicar observaciones, requerimientos, detección de errores, entre otras acciones. Un archivo BCF no contiene información gráfica compleja, sino que se enfoca en la información textual, capturas de pantalla y los identificadores únicos de los elementos asociados. Por esto, los archivos son mucho más livianos que los de modelo en formato nativo, lo que facilita aún más su intercambio.

IDM - Information Delivery Manual: Para que varios actores de distintos ámbitos (público o privado) puedan trabajar en conjunto de forma eficiente, es necesario que todos tengan claridad de cuándo se requerirá o recibirá qué tipo de información, y cómo será este proceso. Esto cobra especial relevancia al involucrar herramientas digitales en la ejecución de los trabajos, ya que algunas de ellas pueden presentar limitaciones o particularidades en la interpretación de la información. El IDM fue creado específicamente para hacerse cargo de esta problemática, proponiendo una metodología de captura y especificación de procesos y flujos de información durante el ciclo de vida de un proyecto ${ }^{72}$.

\subsection{Estándares de la serie ISO 19650}

Las normas de la serie ISO 19650 definen un marco de buenas prácticas para la generación, intercambio y gestión de la información en todo el ciclo de vida de los proyectos de la industria AEC que utilizan BIM en sus procesos. Estos principios son aplicables tanto en proyectos en desarrollo como en edificaciones e infraestructura ya construida, de cualquier tamaño y complejidad, aunque se recomienda que su uso sea criterioso y proporcional. En el contexto de la industria chilena, el Instituto Nacional de Normalización (INN) hizo una adopción directa de las partes 1 y 2 de esta serie, por lo que ahora también son norma chilena. Las normas que son parte de esta serie son:

- La Norma Nch/ISO 19650-1, que define principios y conceptos base para los procesos de generación y gestión de la información.

- La Norma Nch/ISO 19650-2, que establece los procesos de generación y gestión de la información durante la fase de desarrollo de un proyecto.

- La Norma EN-ISO 19650-3, publicada en septiembre de 2019, establece los

\footnotetext{
72 Basado en la definición de buildingSMART International https://technical.buildingsmart.org/standards/information-delivery-manual/ 
procedimientos de gestión y utilización de la información durante la fase de operación de un activo.

- La Norma EN-ISO 19650-5, publicada en junio de 2020, define los principios de seguridad en la gestión de la información.

Ya mencionado anteriormente, a nivel nacional, se cuenta con el Estándar BIM para Proyectos Públicos, desarrollado por Planbim de Corfo publicado en junio del 2019. Este Estándar cumple el rol de anexo nacional de la serie ISO-19650, especificando cómo se debe aplicar esta norma en el contexto local.

Otros estándares relacionados con el desarrollo de proyectos de la industria AEC bajo la metodología BIM son:

- ISO/TS 12911:2012: Framework for building information modelling (BIM) guidance.

- ISO 16757-1:2015: Data structures for electronic product catalogues for building services-- Part 1: Concepts, architecture, and model.

- ISO 16354:2013: Guidelines for knowledge libraries and object libraries.

- NCh-ISO12006-3:2018: Construcción de edificaciones - Organización de la información de los trabajos de construcción - Parte 3: Marco para la información orientada a objetos.

Los estándares ISO referidos a la industria AEC que se encuentran actualmente en desarrollo son:

- ISO/AWI 19650-4: Organization and digitization of information about buildings and civil engineering works, including building information modelling (BIM) -Information management using building information modelling -- Part 4: Information exchange.

- ISO/CD TS 19166: Geographic information -- BIM to GIS conceptual mapping $(B 2 G M)$.

- ISO/DIS 23387: Building Information Modelling (BIM) -- Data templates for construction objects used in the life cycle of any built asset -- Concepts and principles.

- ISO/WD TR 23262: GIS (Geospatial) / BIM interoperability. 
- ISO/WD 22057: Enabling use of Environmental Product Declarations (EPD) at construction works level using building information modelling (BIM).

\section{Discusión y Conclusiones}

El objetivo del documento es incentivar la reflexión en torno a los contenidos abordados en carreras profesionales relacionadas a la industria AEC, y cómo las exigencias del mercado laboral impulsan la renovación de éstos. Desde esta mirada se desarrolla un texto referenciado a la metodología BIM, las potencialidades de tener un entorno colaborativo de trabajo y los estándares que permiten que este proceso se pueda llevar cabo de manera exitosa y eficaz.

De este modo se propone y se cree que es necesario promover el uso de la metodología BIM potenciando el desarrollo del trabajo colaborativo, desde la incorporación de ésta en los programas de estudios en educación superior, para que los futuros profesionales y los ya egresados obtengan las competencias que el mercado está exigiendo.

Será fundamental para los profesionales de la arquitectura, ingeniería y otras carreras relacionadas a proyectos de construcción, obtener las competencias y capacidades respecto a los estándares y tecnologías de entornos comunes de trabajo y el desarrollo de habilidades blandas que permitan la implementación exitosa de una metodología colaborativa, entendiendo que es una herramienta clave en la ejecución eficiente de un proyecto.

En conclusión, fomentar la enseñanza de BIM, ya no solo se debe hacer desde una mirada tecnológica, sino además incrementando el trabajo colaborativo a partir de los estándares asociados como parte del proceso de transformación digital de los futuros profesionales.

\section{Referencias}

Change Agents AEC, BIM Excellence (2016). Matriz de Madurez BIM. v. 1.22. Jul 2016 Matriz reformateada y hecha pública como parte de BIMe Initiative. La versión española ha sido traducida por Víctor Roig de BIMETRIC Laboratorio de Procesos SL.

Eastman, Ch.; Teicholz, P.; Sacks, R.; Liston, K. (2008). BIM. Handbook: A Guide to Building Information Modeling for Owners, Managers, Designers, Engineers and Contractors. John Wiley \& Sons. 
lale Tecnología (2017). Estudio de Identificación de Demanda de Capital Humano con Capacidades BIM en la Industria de la Construcción. Informe Final. Preparado para PlanBim - Corfo.

Organización Internacional de Normalización (2018). Organización y digitalización de la información relativa a trabajos de edificación y de ingeniería civil, incluyendo BIM (ISO 19650-1).

Planbim CORFO (2019). Mibim, Matriz de Madurez Implementación BIM para Organizaciones.

Planbim CORFO (2019). Matriz de Roles BIM. Creación 2018, última actualización junio 2019.

Planbim CORFO. (2019). Estándar BIM para proyectos públicos. Primera edición, junio 2019. Licencia Creative Commons Atribución-NoComercial 4.0 Internacional.

Roig-Vila, Rosabel (2007). Investigación en docencia universitaria. Editorial Octaedro.

Succar, B.; Kassem, M. (2015). Macro-BIM adoption: Conceptual structures. Automation in Construction (57), 64-79.

Palma, R. \& Rossado, V. (2015). Universidad de Lima Perú. Serrano Torres, Daniel Antonio. Safe Solutions Perú. La enseñanza de una metodología integral de colaboración para la gestión de proyectos en construcción civil: la metodología BIM en la educación superior.

Schwab, K. (2016). La Cuarta Revolución Industrial. Random House.

\section{Karen Gutiérrez D.}

Paulina Godoy del C.

Escuela de Arquitectura. Facultad de Arquitectura, Diseño y Artes. 